\title{
A Grid Infrastructure for Supporting Space-based Science Operations
}

\author{
Robert N. Bradford \\ NASA Marshall Space Flight Center \\ Ground Systems Department \\ Huntsville, Alabama \\ Sandra H. Redman \\ University of Alabama in Huntsville \\ Information Technology and Systems Center \\ Huntsville, Alabama 35899
}

\section{Introduction}

Emerging technologies for computational grid infrastructures have the potential for revolutionizing the way computers are used in all aspects of our lives. Computational grids are currently being implemented to provide a large-scale, dynamic, and secure research and engineering environments based on standards and next-generation reusable software, enabling greater science and engineering productivity through shared resources and distributed computing for less cost than traditional architectures. Combined with the emerging technologies of high-performance networks, grids provide researchers, scientists and engineers the first real opportunity for an effective distributed collaborative environment with access to resources such as computational and storage systems, instruments, and software tools and services for the most computationally challenging applications.

The purpose of this study is to provide a basis for implementing grid technology in support of NASA's space based science programs on free flying satellites, the Shuttle and the International Space Station. With the evolution, proliferation and growth associated with computer system and network technologies and the evolution of standards and technologies of ground systems in support of space based operations, a space-based science operations grid can be implemented which will apply the benefits of grid technology to space operations.

In support of space-based science operations, a grid would enable real-time or near realtime access by scientists and engineers to distributed and diverse computational and storage resources that are currently not available to them. Access to this type of computing could have significant advantage to space based science in that principal investigators and spacecraft operators could make real-time or near real-time adjustments to experiments or engineering problems based on high order analysis resulting in more precise and efficient operations and substantial cost-savings. A grid would provide a standard for access to common services in support of space based science operations, including acquisition, processing, and distribution of telemetry; voice communications between control centers, experimenters and users; video flows; commanding of experiments and various types of science and experiment planning. Other advanced 
services common to a grid environment, such as collaboration services, would also be available, creating a more robust environment by integrating the operations and research environments.

With this architecture, substantial savings in the cost of development and operation of space-based experiments can be realized. Instead of a payload developer designing and building unique interfaces for the space science services, the developer would "plug in" to the space-based science operations grid, determine the availability of services and have access to them without needing to know the details of the services being provided. In addition, any other applications required by a researcher for their research could be fully and securely integrated into the same grid architecture, resulting in a collaborative environment rich with capabilities, but low in cost.

\section{Scope}

Although a space-based science operations grid could eventually include all science support centers for Shuttle and ISS payloads and free flying satellites, the scope of this paper is limited to the study of grid technology to support ground operations for International Space Station payloads conducted out of the Payload Operations and Integration Center (POIC) at the NASA Marshall Space Flight Center. Even though the scope of this paper is limited to ISS payloads it should be noted at this time that support for individual legacy satellites may not be feasible. This is mainly because of the age of the legacy satellite and ground equipment, and the budget constraints associated with these older projects that now have limited lifetimes. Also, it is understood that with the limited science currently being conducted on the Space Station, and the limited downlink capabilities of most legacy satellites, especially deep space vehicles (with the possible exception of EOS satellites), there may be no need for on demand high order processing. We assume that this will change as the ISS matures. As this changes, the grid environment can be easily expanded to include these capabilities.

A study to determine the feasibility of implementing a grid to support ISS payload operations out of the MSFC/Payload Operations Integration Center (POIC) is being conducted. Ultimately, the goal is to provide the necessary voice, video, commanding, telemetry and planning services in a secured environment, to support a grid based ISS space-based science operation. The successful application of grid technology to the ISS payload operation would also demonstrate the feasibility of a similar application for NASA's free flying satellites and Shuttle ground science operations, as well as potential control center functions.

\section{Emerging Grid Technology}

"Grids are persistent environments that enable software applications to integrate instruments, displays, computational and information resources that are managed by diverse organizations in widespread locations." A space-based science operations grid could then be defined as a "persistent environment that enables software applications (POIC ground based systems and user interfaces) to integrate (space based scientific) 
instruments, (telemetry) displays, computational and information resources that are managed by diverse organizations (MSFC, GSFC, JPL, and PI) in widespread locations (JSC, KSC, GSFC, MSFC, ARC, JPL, GRC, and remote PI locations)."6

Grids are technology and an emerging architecture that involves middleware that mediate between science portals, applications, and the underlying resources (compute, data, and instrument) in order to simplify the construction of large-scale problem solving systems. Grids provide security, resource management tools, applications, access to remote storage, collaboration services, and distributed processing capabilities in an integrated software environment. Distributed processing capabilities, coupled with the grid enabled, science specific analysis and processing services and access to remote data, provide a portal for any researcher to access discipline specific services without the time consuming and costly "system setup" required today. [Bill Johnston]

The term computational grid comes from an analogy with the electric power grid. The electric power grid brought about a revolutionary change in our lives; it allows us to use geographically distributed resources transparently and consistently. It is ubiquitous, reliable, accessible, coordinated, and inexpensive. [1] The computational grid may well provide a revolutionary change to computing in the same way the electric power grid brought a change to using electrical power. Table 1 provides a grid architecture functional diagram [2]. 

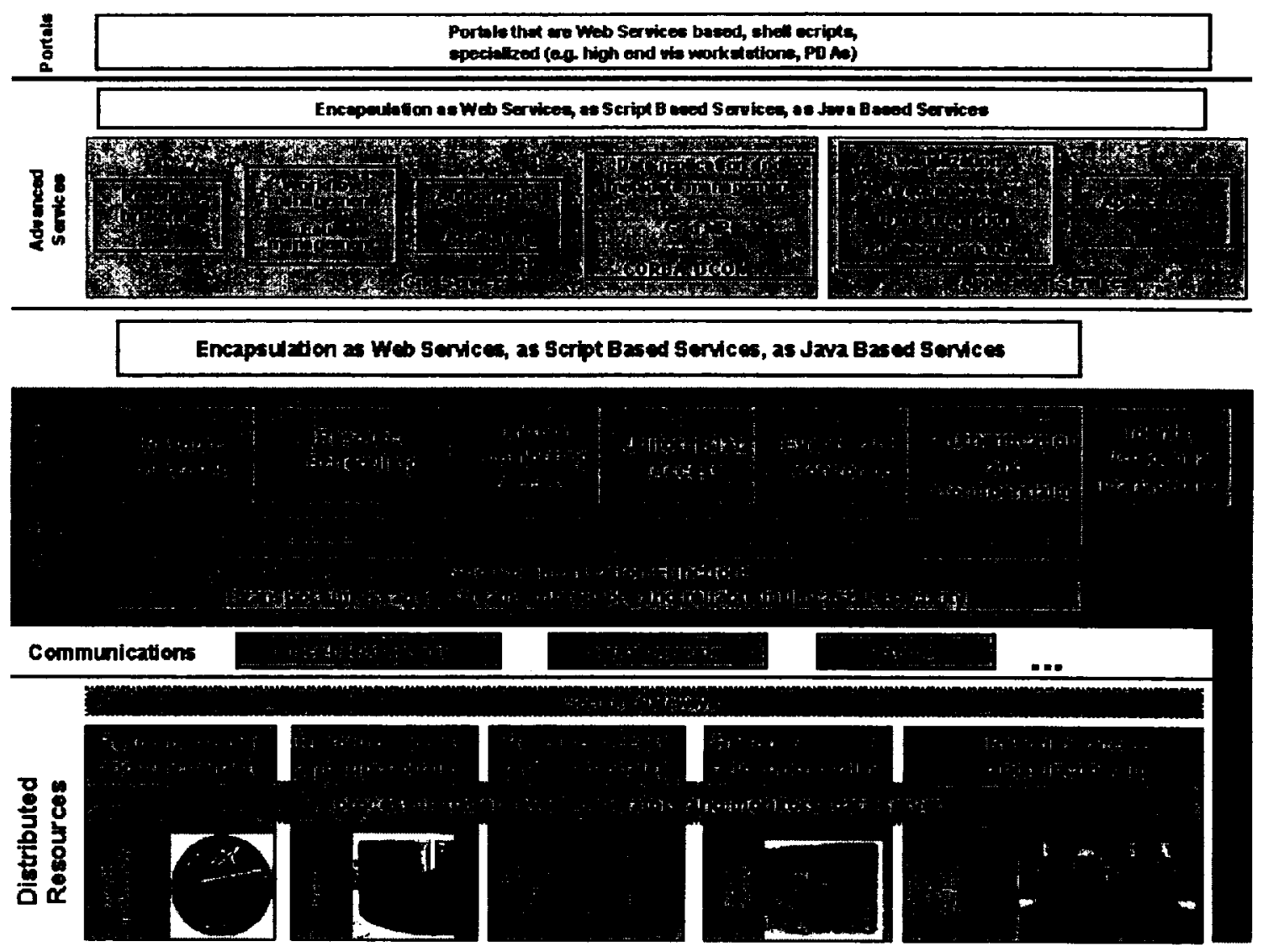

Table 1 A Grid Functional Diagram

\section{Grid Development}

The overarching grid organization is the Global Grid Forum (GGF)

(http://www.gridforum.org/). Composed of representatives from 200 organizations and over 30 countries, the GGF supports the creation and development of global computational grids. The GGF is developing standards and best practices for grid technologies and applications, as well as facilitating the broad deployment of fundamental services for large-scale science and engineering activities worldwide.

The Middleware and Grid Infrastructure Coordination (MAGIC) initiative is coordinating interagency efforts in grid and middleware. Members include NASA, DOE, NSF, NIH, NIST, NOAA, and DARPA, as well as representatives from industry. MAGIC provides a federal voice for international coordination of grid technologies.

A major activity is the National Middleware Initiative (NMI) (http://www.nsfmiddleware.org). Funded by the National Science Foundation (NSF), NMI is developing and deploying a reusable, expandable set of middleware functions and services to benefit collaborative applications in a networked environment. This middleware will allow scientists and engineers to transparently use distributed resources in a collaborative environment. NMI includes two major efforts, NMI-Enterprise and 
Desktop Integration Technologies (EDIT) and the Grid Research Integration Deployment and Support (GRIDS) Center. NMI-EDIT middleware includes directory services, identifiers, authentication, authorization, and Public Key Infrastructure (PKI). See www.nmi-edit.org for an overview of this core middleware. The GRIDS Center is developing middleware, such as the Globus Toolkit, Condor-G and the Network Weather Service, for enabling grid applications. The Globus Toolkit ${ }^{12}$ is an essential part of the grid technology and provides many of the tools needed to implement a grid. It is concerned with three primary areas of grid technology; Resource Management, Information Services, and Data Management. [http://www.nsfmiddleware.org/documentation/GlobusToolkit/] The Condor-G software manages grid resources and job queues. [http://www.nsf-middleware.org/documentation/CondorG/] The Network Weather Service is a distributed, generalized system for producing shortterm performance forecasts based on historical performance measurements [http://www.nsf-middleware.org/documentation/NWS/]

The Open Grid Services Architecture (OGSA) is the proposed standard for the integration of grid and web services concepts and technologies. Still under development, OGSA evolves the Globus Toolkit using concepts from both the grid and web services communities. http://www.globus.org/ogsa/. The goal of OGSA is to provide standardsbased uniform integrated services for disparate resources while providing the needed QoS for applications. Creating, naming, and discovering transient grid services instances, location transparency and multiple protocol bindings for services instances, and support for integration with underlying native platform facilities are provided for by OGSA. OGSA uses the Web Services Description Language (WSDL) interfaces and associated conventions to define mechanisms required for creating and composing sophisticated distributed systems and the management of those resources. [6]

Table 2 identifies several on going grid implementations.

- NASA Information Power Grid

- TeraGrid - Distributed Terascale Facility

- GriPhyN - Grid Physics Network

- iVDGL - International Virtual Data Grid Laboratory

- BRN - Biomedical Informatics Research Network

- PPDG - Particle Physics Data Grid

- DATATAG - Trans-Atlantic Grid Testbed

- NEES - Network for Earthquake Engineering Simulation

- AstroGrid - UK Virtual Astronomy

- sciDAC - DOE Science Grid

- Nauka Grid - Russia Science Grid

- NorduGrid - Norway Science Grid

- APGrid - Asia Pacific Grid

Table 2 - Established Grids 


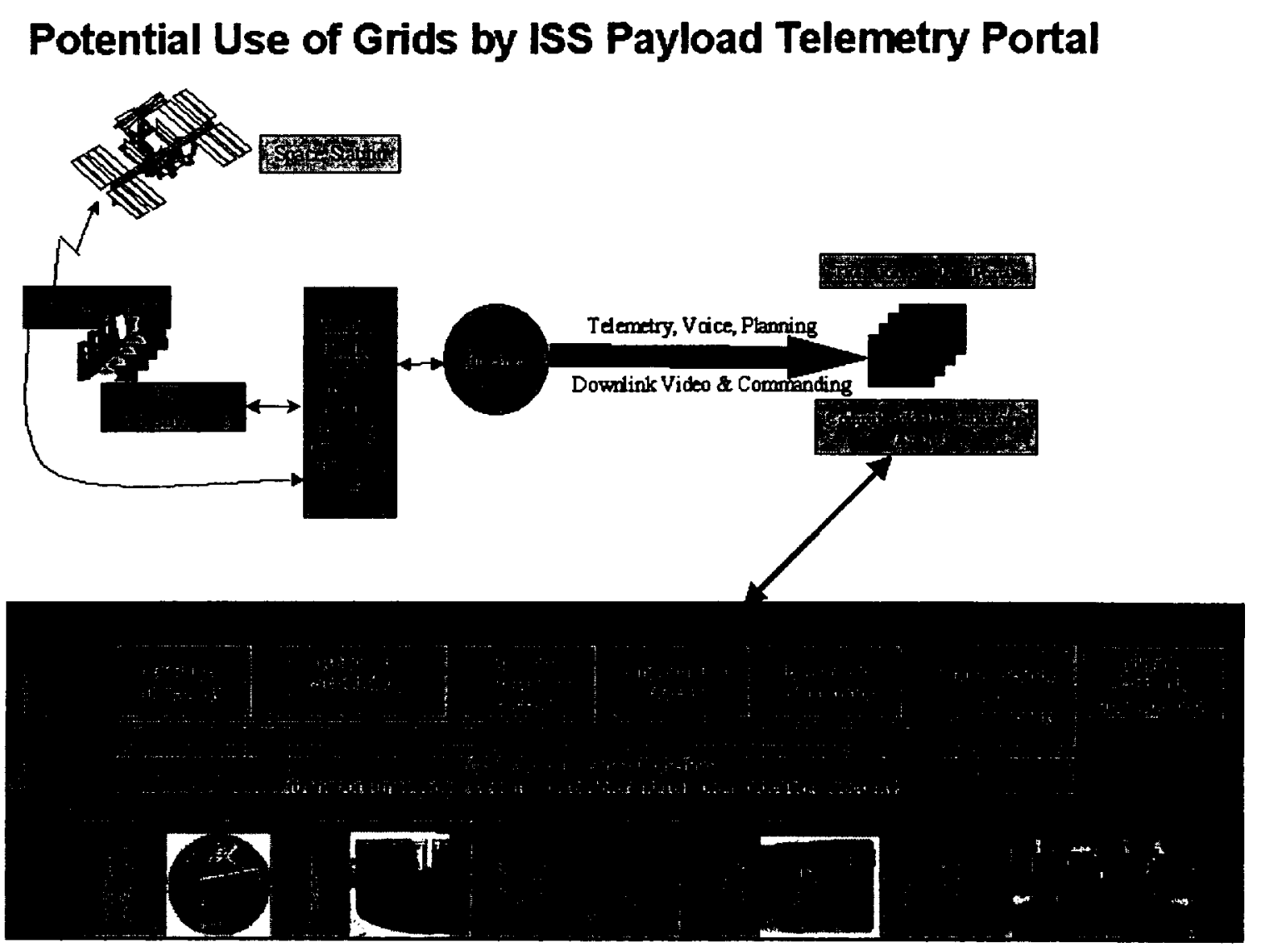

Methodology and Approach to Developing a Space-based science operations grid

The approach used in this study is to detail the current method of conducting space operations and determine the necessary services required for the development of a Spacebased science operations grid for ISS payload operations. Existing technologies and standards used for payload operations will be identified, as well as identifying existing tools, technologies and standards that lend themselves to a Space-based science operations grid. At a high level, requirements for porting these applications to the grid environment will be defined. For clarity, examples will be provided whenever possible. Needed components to developing a space-based science operations grid will also be identified. Finally, the systems, standards and networks that need to be further enhanced or which need to be developed to support space science operations will be discussed.

\section{Defining the Space-based science operations grid}

To successfully implement a space-based science operations grid, it is necessary to identify the required services and other necessary technologies required for space science operations. These are the services that will make up a space-based science operations grid virtual organization. "In a grid the OGSA (Open Grid Services Architecture) defines a Grid service-a Web service that provides a set of well-defined interfaces and that 
follows specific conventions." 11 Current services provided for ISS Payload Operations are defined below.

\section{POIC Space Operations}

The POIC is the single point of control for ISS payload operations. The systems described below provide the necessary services that allow scientists to interact with their experiments. These systems are centralized at the POIC but are web based for remote science operations

- Payload Data Services System (PDSS):

The PDSS acquires, stores, and distributes ISS telemetry to the EHS for POIC cadre, international partners (IPs), Telescience Support Centers (TSCs) and specific principal investigator sites. The PDSS is the telemetry flow interface for all science from ISS.

- Enhanced Huntsville Operation Support Center (HOSC) System (EHS): The EHS performs command processing, real-time and near-real-time telemetry acquisition, processing, and distribution, database services, and system monitor and control services for pre-launch integration and checkout, simulation training and flight operations. EHS provides the operational services required for the Payload Operations Integration Function (POIF) cadre located in the POIC.

- Payload Planning System (PPS):

The PPS provides a set of software tools to automate planning and schedule and analyze payload activities. The PPS uses a web-based interface.

- Internet Voice Distribution System (IVODS).

The IVODS service Internet voice service to the ISS remote scientist via the Internet2/Abilene Network.

\section{Remote Services: TReK, IVoDS and the ERIS Interface}

\section{TReK}

The Telescience Resource Kit (TReK) is a suite of PC-based software applications that can be used to monitor and control a payload on board the International Space Station (ISS). TReK provides both local ground support system services and an interface to utilize remote services provided by the Payload Operations Integration Center (POIC), including: 
- Telemetry

TReK receives, processes, records, forwards, and provides playback of science telemetry and provides exception monitoring (monitoring incoming data for out of range exceptions) of telemetry.

- Commanding

TReK will send a command to your payload, track the command, Command Track (History of most recent commands sent from TReK System) as well as command session recording and viewing (record and view all command activity -commands sent and command responses received).

- Displays

TReK provides the front end to allow a user to program their unique displays in $\mathrm{C}++$ or use the application programming interface. The TReK API provides a way for you to access telemetry functions, to build data displays, computations, and scripts using their various COTS products i.e. Labview.

- Database

One of the more difficult aspects of space operations is determining and defining the database format of the telemetry to be received by the POIC and TReK (telemetry processing information) and the format of the commands to be sent to the POIC for uplink to an individual experiment, Command Database (command processing information). TReK provides a capability to make these definitions within the TReK itself.

- Training, Test \& Checkout

TReK has a component that allows a user to provide stand-alone testing and training to their operations personnel using the simulation function of TReK. Using the defined databases describe above an experimenter without leaving their lab can test their displays, computations, scripts, and commands using the Suitcase Simulator and TReK. The Suitcase Simulator simulates the on board systems for ground test interfaces.

- EZStream®

EZStream is a system that allows a scientist to develop displays without the need for programming support i.e. $\mathrm{C}$ programmers. It provides an interface for access to science telemetry from TReK to the web at a user's location. EZStream was developed under a Small Business Innovative Research contract by AZ Technology. The EZStream system specifications can be viewed at www.aztechnology.com

\section{IVODS}

The Internet Voice Distribution System (IVoDS) provides audio communications to remote users via Internet Protocol (IP) based communications. This multi-conferencing 
system links researchers, NASA Operations Personnel, and ISS crew together to support collaboration during Space Station experiment operations \& planning. Figure 6 shows the capabilities of the IVODS mission voice system.
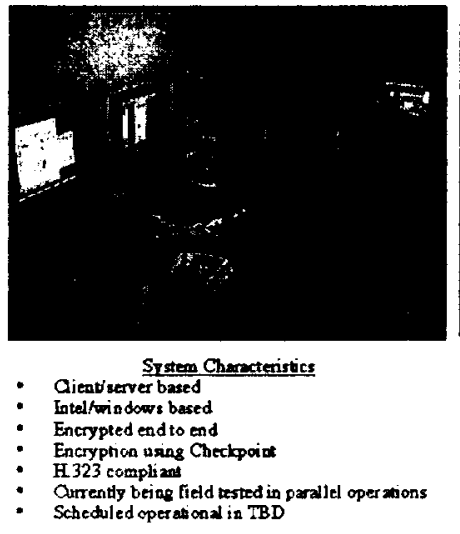

Scheculed oper tional in TBD

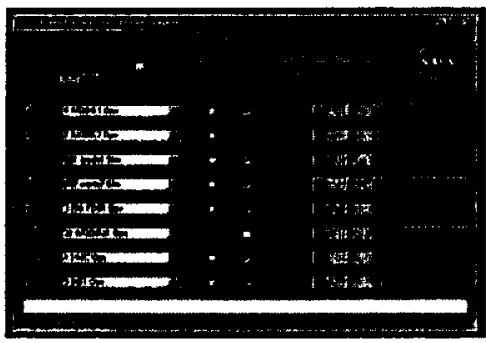

Cempilities

Mositor 8 conferences vimulhmeoxsly, talk on one Tser selects from mithosized rubset of avilable Voice coof trences

Volume contsol/mute for individusl conferences Assign talk and monitor privileges per user

Talk to creso on Space (Air) to Ground if enabled by PAYOOM

inoos sum

Figure 6, IVoDS Overview

\section{ERIS}

The EHS Remote Interface System (ERIS) allows user access to the services provided by the POIC ground systems from the world wide web.

Implementing the Space-based science operations grid

For the purposes of this paper, there are seven components to a space-based science operations grid; 1) Security, 2) Payload/Experiment Control Services, 3) Network, 4) Remote User/Instrumentation Portal, 5) Education and Public Outreach, 6) Collaboration, and 7) Integration of Custom Applications.

Component One - Security: This component is an overarching service that is of vital importance to the successful grid operation. This service, composed of many subparts, is required to be implemented end to end and is commensurate with the user operational concept resident at that site. In other words, if a user (and therefore the site) has command privileges then the security end to end must be commensurate for the protection of the command functions. For example, encryption is employed end to end for voice, commanding and planning data for ISS payloads. It is assumed that individual experiment(s) telemetry is encrypted by the user within the experiment to the ground and through the grid. 


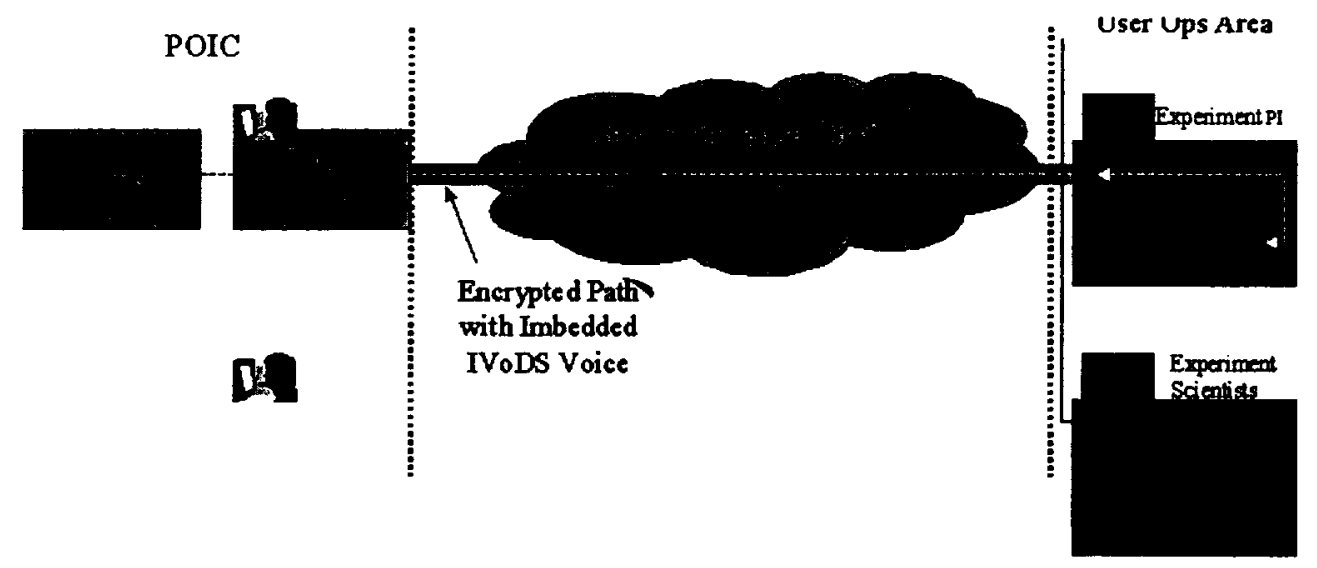

Figure 1, IVoDS Security

Component Two - Payload/Experiment Control: Payload and experiment science operations are considered a prime candidate for grid services within this component. Both PC and web based science operation at MSFC's Payload Operations Integration Center (POIC) is considered potentially conducive to grid operations. These services would include telemetry, commanding, voice, video, and planning

Component Three - Network: This component is made up of all of the available networking infrastructures now associated with the communications industry. Local area networks, first and last mile networks, and wide area networks including private and public networks are included. One factor that enables grid implementation is the robust connectivity that exists today. Figures 2 and 3 shows examples of the worldwide connectivity associated with the Research and Education Networks (RENs) that will be the network solution for the space-based science operations grid.

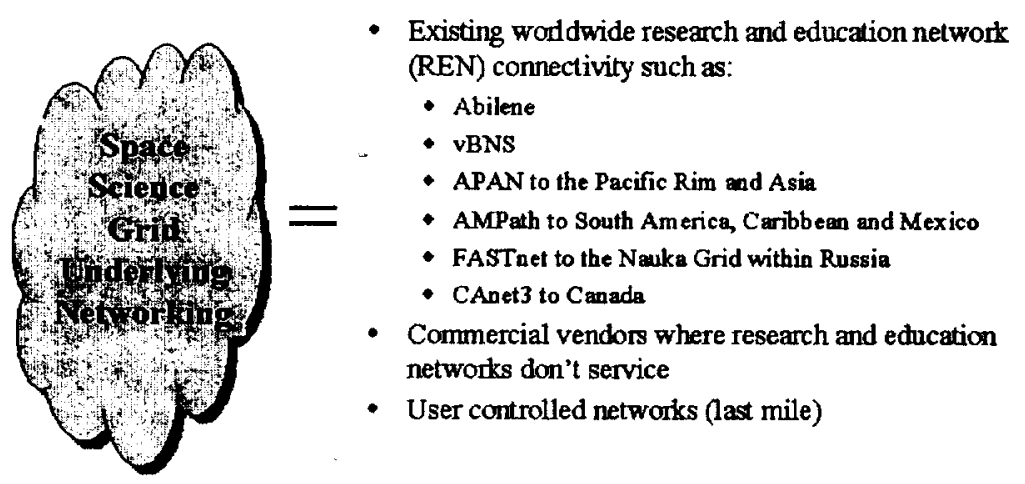

Figure 2, Underlying Space-based Science Operations Grid Network 


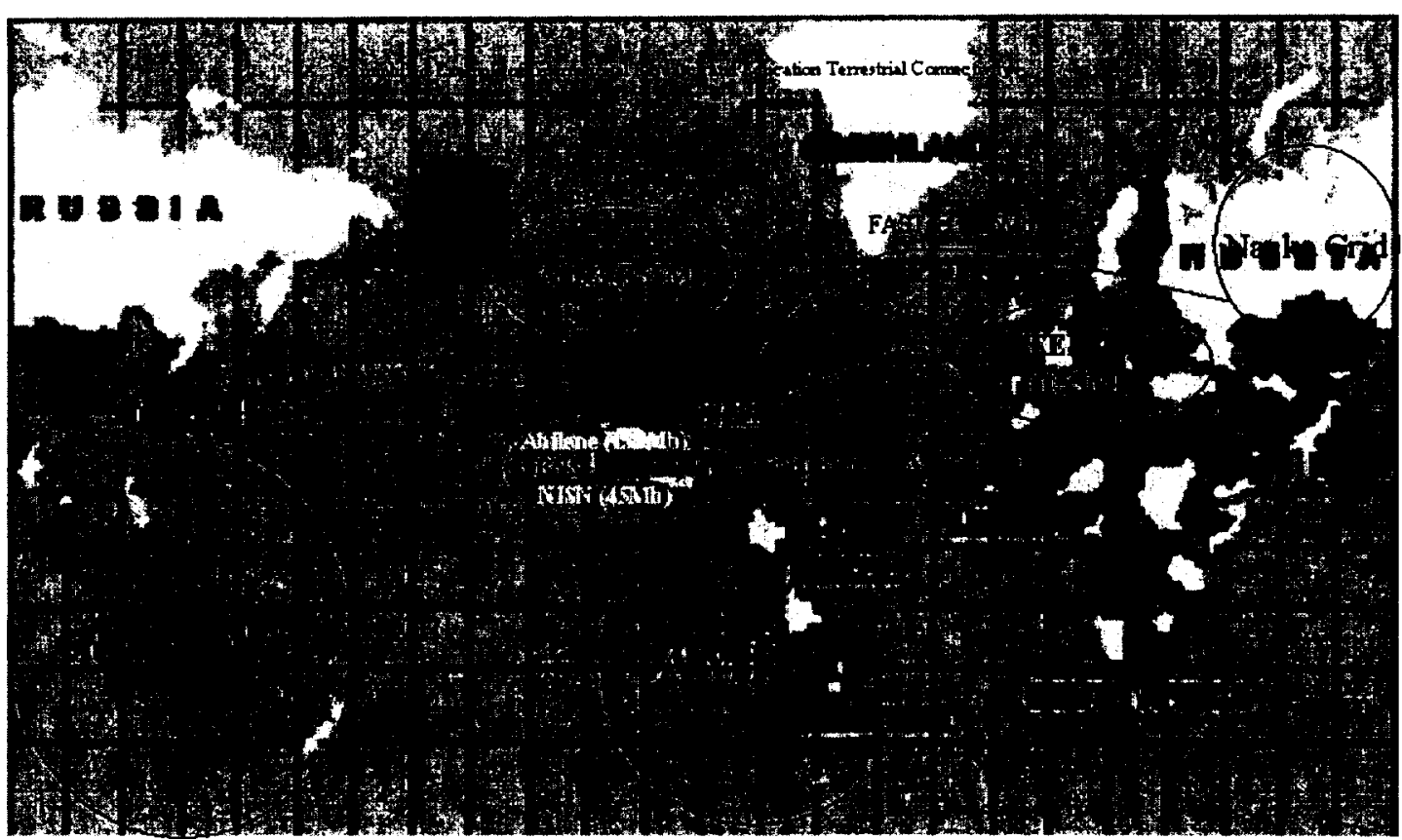

Figure 3, Worldwide Underlying Network

Networks within a national boundary are prevalent except in Africa and the Mideast. Networks such as Abilene, which is the Internet 2 research and education network for the United States, provide extensive connectivity within national boundaries. Figure 4 shows Abilene connectivity for the USA. As indicated there are multiple peering arrangements between various networks that provide near limitless connectivity.

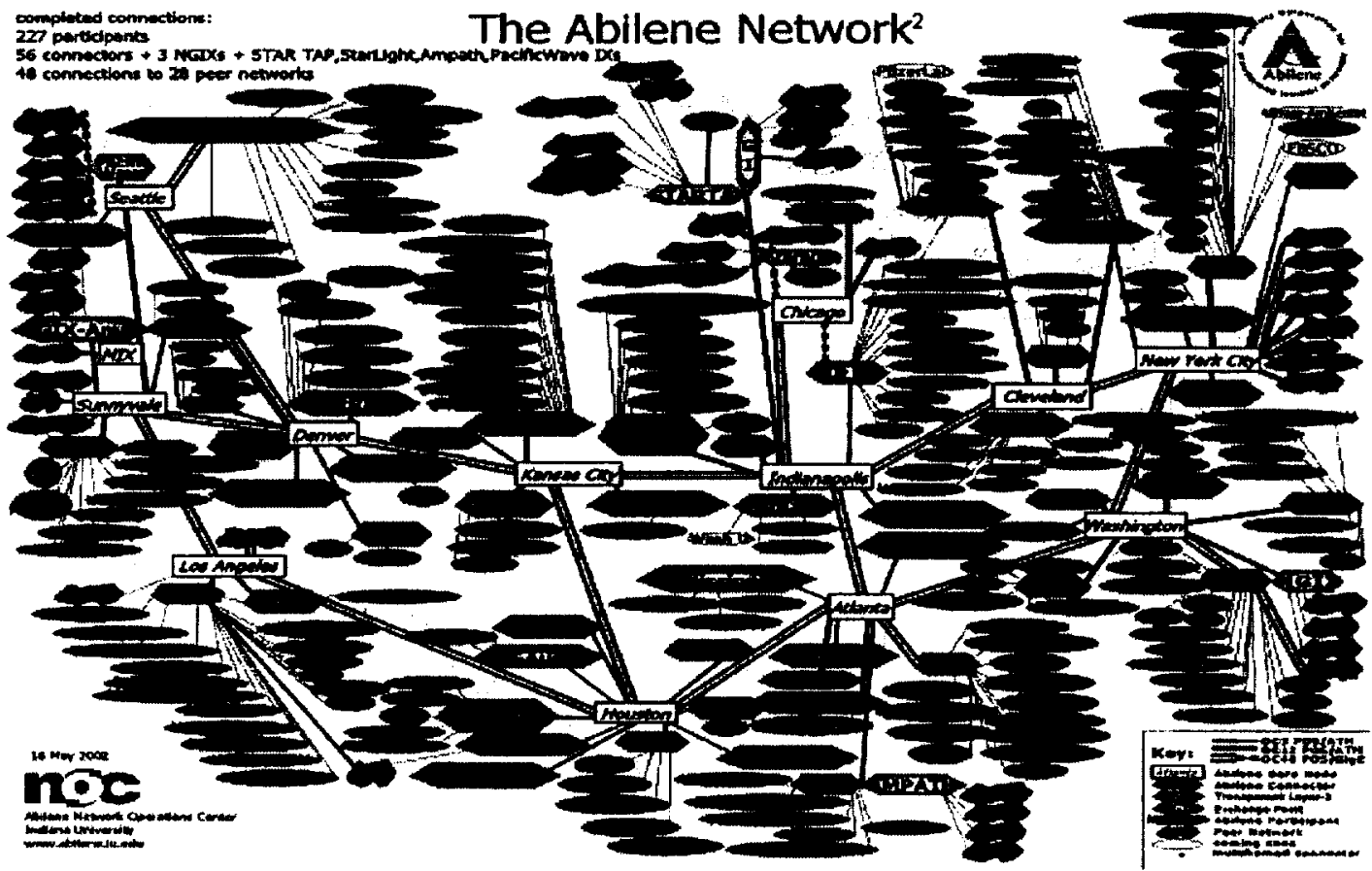

Figure 4, Connectivity for the Abilene Network 
Networking is considered a vital part of the Grid for obvious reasons. With the advancement of the Internet Protocol (IP) over almost any media, i.e. ATM, frame relay and satellite based networking, the network has become one of the least problematic areas in terms of implementing a space-based science operations grid. Although there are issues associated with networks, such as security and QoS for real-time type operations, there is a considerable combined and coordinated effort to resolve these issues.

NASA's four levels of Internet Protocol services are depicted in Table 3. Within these four levels, NASA uses three data points to measure the performance of a network used to support space based missions. They are packet loss, availability and latency.

\section{- Performance Measurements}

- Roundtrip Latency, Availability (Reachability) \& Packet Loss

- NASA's Mission Network Specifications

\begin{tabular}{|c|c|c|c|c|c|c|}
\hline & $\begin{array}{l}\text { Service } \\
\text { Category } \\
\end{array}$ & Avaibaility & $\begin{array}{l}\text { Restoral } \\
\text { Time }\end{array}$ & $\begin{array}{l}\text { Coverage } \\
\text { Period }\end{array}$ & $\begin{array}{l}\text { Acreptable } \\
\text { Packet Loss }\end{array}$ & $\begin{array}{l}\text { Round Trip } \\
\text { Time }\end{array}$ \\
\hline \multirow{3}{*}{ Critical } & $\begin{array}{l}\text { Real-time } \\
\text { Critical }\end{array}$ & $99.98 \%$ & $<1$ min & $24 \times 7$ & $0.001 \%$ & $<120 \mathrm{~ms}$ \\
\hline & $\begin{array}{l}\text { Mission } \\
\text { Critical }\end{array}$ & $99.95 \%$ & $2 \mathrm{hr}$ & $24 \times 7$ & $0.001 \%$ & $<120 \mathrm{~ms}$ \\
\hline & Premium & $99.50 \%$ & $4 \mathrm{hr}$ & $24 \times 7$ & $<1.0 \%$ & $<100 \mathrm{~ms}$ \\
\hline Non-Critical & Standard & $99.50 \%$ & $<24 \mathrm{hr}$ & $\begin{array}{l}\text { Gom Eastem } \\
\text { Monday to } 6 \\
\text { p.m Pacific, }\end{array}$ & $1.00 \%$ & $<250 \mathrm{~ms}$ \\
\hline
\end{tabular}

Table 3, NASA's Network Performance Specifications

In a study conducted in May 2002, the NASA specification for latency was universally met. [15] The measurements for packet loss and availability were not met. In an ongoing study, the public network performance between NASA sites is being conducted to determine whether public/private network performance is adequate to support a space operations grid. With the evolution of networking technology with guarantee of delivery these specifications may be revisited.

Component Four - Remote User/Instrumentation Portal: This component is made up of the ground-based systems that interface with the POIC from an experimenter's location. This component is the portal from which an experimenter would gain access to the necessary operational services they need to command and control their experiments. The services in this component provide an end-user the ability to receive, process, analyze and display telemetry, command experiments or instruments including command management activities, i.e. command response from the end-user site. There are several 
current systems that are used in support of ISS science that have applicability to this component. As shown in Table 3 there are many current systems that have been identified as candidates for use in a space-based science operations grid.

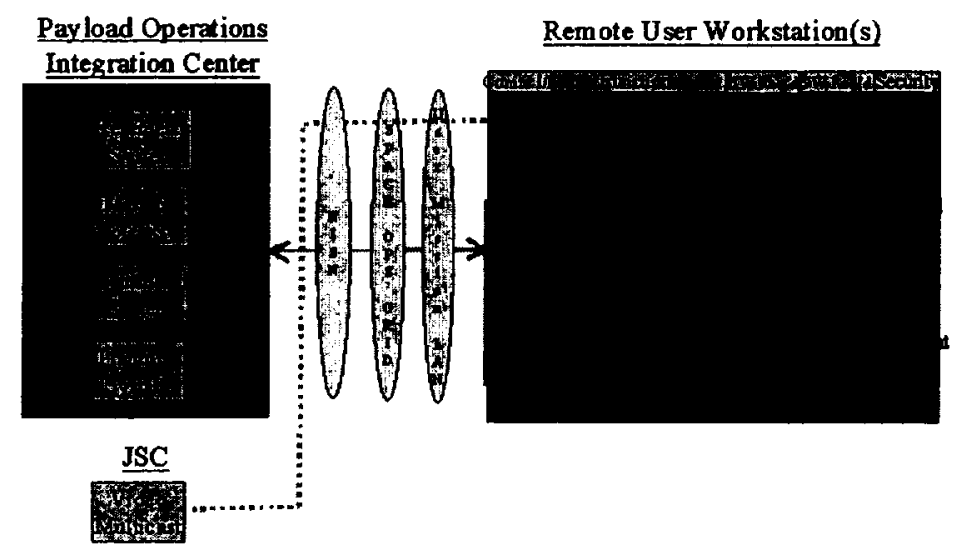

Figure 5, TReK End to End

For more information on these systems see the web site section at the end of this paper. The interface for TReK is unique to ISS but is currently (as of July 2002) being updated to provide a more robust connectivity i.e. TDM. Figure 5 shows the TReK in an end-toend configuration for ISS payloads.

Component Five - Education and Public Outreach: This component is not necessary for space science operations per se but all NASA programs have an educational and public outreach component. The nature of education and outreach is highly distributed but not necessarily processor intensive. The distributed nature makes it a candidate for the space-based science operations grid where a school or school system becomes a node with a profile on the grid.

Component Six - Collaboration: This component brings together all the tools and services that allow interactive collaboration in the distributed, heterogeneous grid environment. The ability to collaborate in the same environment that supports science operations, with collaborators that may be using different platforms, in different locations, provides more effective environment for research.

Component Seven - Integration of custom applications: This grid component, which is not provided in the payload operations systems of today, provides perhaps the most significant value-added service to the space-based science operations grid concept. Scientists or engineers will be able to integrate custom applications to the grid environment using standards based mechanisms. A single environment for the scientist or engineer to perform research will provide substantial cost-savings for hardware, software, and labor. 
Table 4 shows the systems that provide the services described above. Many of these systems are currently web based and are considered ideal candidates to enable a spacebased science operations grid implementation.

Services and (Potential Solutions) by Grid Portal Component:

\section{Component}

1. Security Component

- PKI

2. Payload/Experiment Control

- Telemetry processing, distribution EHS

- Command processing EHS

- Database Services EHS

- Display Services EHS

- Telemetry acquisition and distribution PDSS

- Payload planning PPS

- VoiceDistribution IVODS

3. Network Component

4. Remote User/Instrument Portal Component

- Mission voice incluating air to ground and space to ground biniss

- ISS Video

- Commanting and command data basing

- Tel emetry processing and data clisplay

- Planring and re-planting

5. Educational and Public Outreach Component
Potential Solution

(Imbedded at the system level)

Abilene

IVODS

IViDS requires derd opment

TReK, Web, EPC

TReK, Web, EPC

Web, Rose \& other TBD systems

(EZStream(B)

Table 4, Possible Existing Service Solutions by Space-based science operations grid Component

Figure 8 shows at a high-level an example of a grid in support of the ISS payload community.

Space Science Grid Concept with Some

Specificity (Cont)

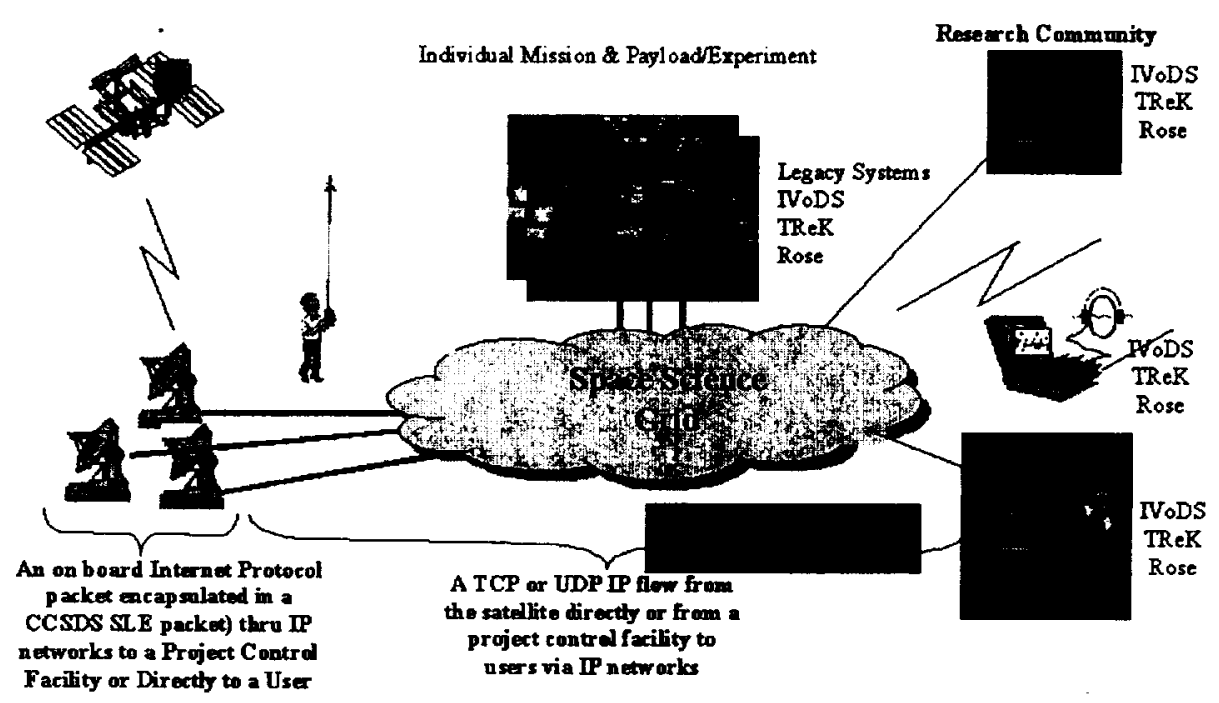


Figure 8, Example of a Space-based science operations grid for ISS Payloads

\section{Future Directions in grid technology for Space Based Science Operations}

The space based science operations grid can be greatly expanded. One of the single most important factors in space operations is the space to ground interfaces for the downlink of telemetry and the uplink of commands and other data. A Vehicle Component to could be added the grid concept, enabling the experiment itself to be a grid node, when IP is used end-to-end. With the vehicle a grid node, more flexibility and efficiency could be realized as older and more costly technology is phased out. The Vehicle Component would be comprised of free flying satellites, Shuttle science payloads and ISS experiments.

Another component should be added to include a Mission Control Component. This component would provide for the safe operation of the vehicle. An experiment can be controlled by commands originating from a ground based control center like the Payload Operations Integration Center (POIC) or a remote experimenter's location. Services provided in this component would include flight dynamics, commanding, resource utilization and on board health and safety including monitoring mission success criteria. As a grid service, applications such as TreK and IVoDS can be used in a control center environment without significant change, providing a cost effective solution. Under the science umbrella, there are existing systems at NASA centers that are specifically designed for specific missions/programs. Some of these systems are dated and may not be candidates for a grid implementation.

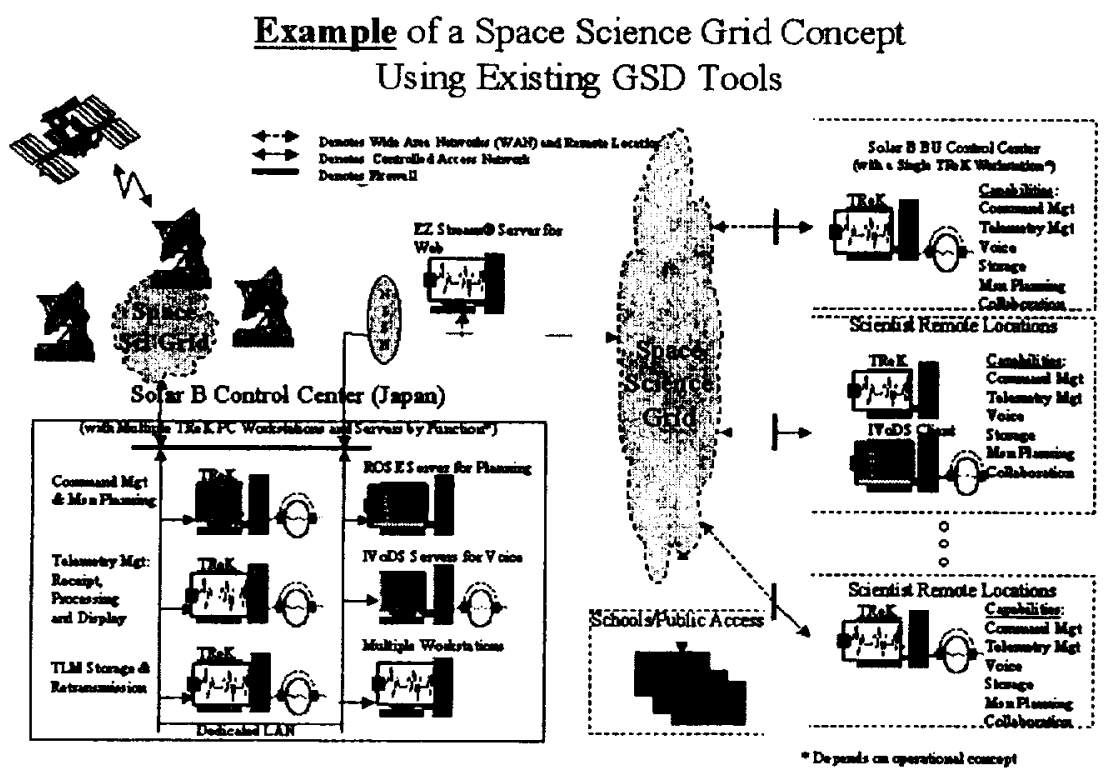

Figure 7, Example of a Space based Science Operations Grid for a Free Flying Satellite 
Figure 7 shows an example of a Space-based science operations grid for the Solar B Satellite. As depicted in Figure 7 all users and control center operators are nodes on the grid. Essentially all locations would have equal access unless otherwise specified in profiles associated with each site/user depicting which services a user can access. Under this example all the services, except Flight Dynamics, are represented by the systems listed in Table 4. The Space-based science operations grid cloud in Figure 7 may be comprised of various research and education networks at the state leve, i.e. Alabama Research and Education Network (AREN), nationally, i.e. Abilene or internationally, i.e. AMPath. And as shown in the example, the underlying network may include government and/or private networks.

\section{Conclusion}

The implementation of a space-based science operations grid to support ISS payloads is feasible. This is based on the existence of a network infrastructure and supporting middleware that are conducive to grid technology in general and space operations in particular. The space-based science operations grid will provide a standard interface to services, access to greater computational and data storage resources, and provide an architecture that is scalable and that can be expanded in concept and practice. And, implementation risks for a space-based science grid are reduced since several web based systems that provide many POIC services are already in existence.

The cost saving factors associated with implementing a space-based science operations grid are many. A grid can use existing network connectivity and bandwidth, and share network resources with other applications and technologies. Network technology has evolved to the point that Gigabit transmissions are now common. Use of existing networks will allow NASA to avoid high cost dedicated circuits/services. Existing systems developed to support ISS, and ported for grid use, provide most of the operational and experiment services required for payload operations. The use these systems will help to avoid development costs in the future. Ported to the grid, applications such as TReK, with its robust command features, can also reduce cost for other projects or programs that have commanding and telemetry requirements. The development of grid technology and standards that is sponsored by federal agencies, research universities, and international forums, applies directly to a NASA space-based science operations grid implementation without significant cost to NASA.

A space-based science operations grid will enable wide spread participation in space based science and technology development by a much wider spectrum of the population to include the non-scientific population. Because grids allow access to super computing type processing and dispersed data storage, more science can be provided because of the efficiencies that would be gained. Generally, with a lower cost of implementation of the grid architecture associated with using existing ISS systems, more science can be funded with the cost savings/avoidance.

The future of a space based science operations grid may well be determined by the ease of use and lowering of the cost of ground support systems and processes in support of ISS 
and other NASA missions. The integration of grid services currently provided by applications such as TREK, ERIS, and IVODS will verify that these systems or like systems will provide the services required to operate on a grid. A prototype grid implementation based on the ISS payload community is currently being studied. A collaboration between the Ground Systems Department, the University of Alabama in Huntsville and the Ames Research Center Information Power Grid organizations is being formed to implement the prototype. This prototype will provide proof-of-concept and will help in determining the costs associated with implementing the space-based science operations grid. 


\section{References}

[1] I. Foster and C. Kesselman, "The Grid: Blueprint for a New Computing Infrastructure," 1999, chapter 2.

[2] B. Johnston, "Thoughts on Grid Architecture," Global Grid Forum 4, Feb. 19, 2002

${ }^{12}$ I.Foster and C. Kesselman, "Globus: A Toolkit-Based Grid Architecture," The Grid: Blueprint for a New Computing Infrastructure, I. Foster and C. Kesselman, eds., Morgan Kaufmann, San Francisco, 1999, pp 259-278

"POIC Capabilities Document, International Space Station Program," Space Station Program 50304 Revision B, March 2002.

10 I. Foster, C. Keselman, and S. Tuecke, "The Anatomy of the Grid, Enabling Scalable Virtual Organizations", 2001, p. 10.

11 I. Foster, C. Kesselman, J. Nick, S. Tuecke, “Grid Services for Distributed System Integration," June, 2002, p. 41.

12 I. Foster, C. Kesselman, J. Nick, S. Tuecke, "The Physiology of the Grid: An Open Grid Services Architecture for Distributed Systems Integration," June 22, 2002.

[1] NASA Integrated Services Network (NISN) Services Document, Revision 3-C, November 2000 , pp. $29-30$

[6] I. Foster, C. Kesselman, J. Nick, S. Tuecke, “ The Physiology of the Grid: An Open Grid Services Architecture for Distributed Systems Integration,” June, 2002, p. 1.

[15] R. Bradford, S. Redman, “ 
Applicable Web Sites

[3] Internet2 Abilene network,

http://www.internet2.edu/abilene

NASA Information Power Grid

www.ipg.nasa.gov

Grids Research Integration and Deployment Center

http://www.grids-center.org

The Globus Project

http://www.globus.org

Globus Project Information on OGSA

http://www.globus.org/ogsa/ 


\title{
Technology for a NASA Space-based Science Grid Portal
}

\author{
Space Science 2002 Conference \\ Ground Segment Engineering And Architectures Session
}

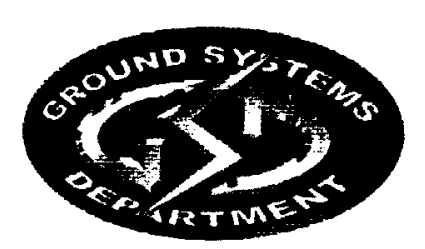

Robert N. Bradford

Marshall Space Flight Center

Flight Projects Directorate

Ground Systems Department
October, 2002

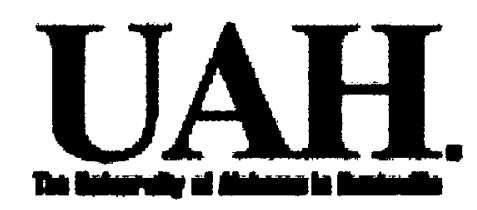

Sandra H. Redman

Information Technology and Systems Center

University of Alabama at Huntsville 


\section{Purpose of Study}

- Hypothesis: With the evolution, proliferation and growth associated with computer system and network technologies and the evolution of standards and technologies of ground systems in support of space based science operations, a Space Science Grid Portal can be implemented which will apply all the benefits of grid technology to space based systems.

- Provides a dynamic, secure and scalable architecture based on standards and next-generation reusable software

- Enables greater science collaboration and productivity through shared resources and distributed computing 


\section{Purpose of Study}

- Hypothesis: With the evolution, proliferation and growth associated with computer system and network technologies and the evolution of standards and technologies of ground systems in support of space based science operations, a Space Science Grid Portal can be implemented which will apply all the benefits of grid technology to space based systems.

- Provides a dynamic, secure and scalable architecture based on standards and next-generation reusable software

- Enables greater science collaboration and productivity through shared resources and distributed computing 
- Space Science Grid Portal as defined by this paper includes the on board ISS payloads and experiments to the scientist located at various universities nationwide

- Includes the necessary grid enabling technologies, networking infrastructure, control systems, tools and end user ground systems 
- Space Science Grid Portal as defined by this paper includes the on board ISS payloads and experiments to the scientist located at various universities nationwide

- Includes the necessary grid enabling technologies, networking infrastructure, control systems, tools and end user ground systems 


\section{Objectives, Methodologies and Approaches}

- Objective:

- Initially, determine the feasibility of implementing a Space Science Grid Portal to support NASA space based science operations

- Ultimately but not a part of this initial activity, provide the necessary voice, video, telemetry services and planning services including security to support free flying satellites, Shuttle based science and the International Space Station ground science operations located at various telescience support centers (TSCs) and other remote user locations

- Methodology:

- Identify existing systems, standards and networks which can fulfill the space based science services listed above, on which ISS payload operations are dependent

- Conduct an examination of current Grid implementations, technology developments

- Measure network performance between NASA site cities to determine if network performance levels are adequate to support NASA real-time operations 
- Objective:

- Initially, determine the feasibility of implementing a Space Science Grid Portal to support NASA space based science operations

- Ultimately but not a part of this initial activity, provide the necessary voice, video, telemetry services and planning services including security to support free flying satellites, Shuttle based science and the International Space Station ground science operations located at various telescience support centers (TSCs) and other remote user locations

- Methodology:

- Identify existing systems, standards and networks which can fulfill the space based science services listed above, on which ISS payload operations are dependent

- Conduct an examination of current Grid implementations, technology developments

- Measure network performance between NASA site cities to determine if network performance levels are adequate to support NASA real-time operations 


\section{Objectives, Methodologies and Approaches (Cont)}

- Approaches:

- Use the Internet Protocol end to end (except for ISS)

- Identify and use existing standards, systems and software

- Where existing systems, standards and software do not fully meet the service requirements, reassess the NASA requirement for relevancy i.e network packet loss

- Assumptions:

- Only one solution need be identified to fulfill a specific service requirement i.e. voice and IVoDS or one adequate network to be considered a feasible solution

- No network link encryption is provided by vendor network providers

- Intrusion detection and virus scanning and elimination is provided at the local level by the remote user or control center 


\section{Objectives, Methodologies and Approaches (Cont)}

- Approaches:

- Use the Internet Protocol end to end (except for ISS)

- Identify and use existing standards, systems and software

- Where existing systems, standards and software do not fully meet the service requirements, reassess the NASA requirement for relevancy i.e network packet loss

- Assumptions:

- Only one solution need be identified to fulfill a specific service requirement i.e. voice and IVoDS or one adequate network to be considered a feasible solution

- No network link encryption is provided by vendor network providers

- Intrusion detection and virus scanning and elimination is provided at the local level by the remote user or control center 
- High Level Definition of a Grid: "Grids are persistent environments that enable software applications to integrate instruments, displays, computational and information resources that are managed by diverse organizations in widespread locations." (Source:

- Grids provide several levels of service

- Provide the identification and location of services on a Grid

- Network service including reserving bandwidth

- Access to remotely located multiprocessor processing based on individual science requirements

- Access to collaborative tools e.g. application sharing, video teleconferencing

- Scheduling of these services

- Definition of a Space Science Grid Portal Under the Defined Scope: A Space Science Grid Portal is a "persistent environment that enable software application (ground based systems e.g. POIC) to integrate (space based scientific) instruments, (telemetry) displays, computational and information resources that are managed by diverse organizations (like the POIC) to widely dispersed locations (e.g. University of Wisconsin, Harvard, University of Colorado)." 
- High Level Definition of a Grid: "Grids are persistent environments that enable software applications to integrate instruments, displays, computational and information resources that are managed by diverse organizations in widespread locations." (Source: )

- Grids provide several levels of service

- Provide the identification and location of services on a Grid

- Network service including reserving bandwidth

- Access to remotely located multiprocessor processing based on individual science requirements

- Access to collaborative tools e.g. application sharing, video teleconferencing

- Scheduling of these services

- Definition of a Space Science Grid Portal Under the Defined Scope: A Space Science Grid Portal is a "persistent environment that enable software application (ground based systems e.g. POIC) to integrate (space based scientific) instruments, (telemetry) displays, computational and information resources that are managed by diverse organizations (like the POIC) to widely dispersed locations (e.g. University of Wisconsin, Harvard, University of Colorado)." 


\section{Grid Development and Coordination}

- GGF (Global Grid Forum) - composed of representatives from 200 organizations and over 30 countries, facilitates and supports the creation and development of global computational grids and is developing standards and best practices for grid technologies and applications

- NMI (National Middleware Initiative) - developing and deploying a reusable, expandable set of middleware functions and services that benefit applications in a networked environment 


\section{Grid Development and Coordination}

- GGF (Global Grid Forum) - composed of representatives from 200 organizations and over 30 countries, facilitates and supports the creation and development of global computational grids and is developing standards and best practices for grid technologies and applications

- NMI (National Middleware Initiative) - developing and deploying a reusable, expandable set of middleware functions and services that benefit applications in a networked environment 


\section{Other Grid Development Activities}

- NASA Information Power Grid

- TeraGrid - Distributed Terascale Facility

- GriPhyN - Grid Physics Network

- iVDGL - International Virtual Data Grid Laboratory

- BIRN - Biomedical Informatics Research Network

- PPDG - Particle Physics Data Grid

- DATATAG - Trans-Atlantic Grid Testbed

- NEES - Network for Earthquake Engineering Simulation

- AstroGrid - UK Virtual Astronomy

- sciDAC - DOE Science Grid

- Nauka Grid - Russia Science Grid

- NorduGrid - Norway Science Grid

- APGrid - Asia Pacific Grid 


\section{Other Grid Development Activities}

- NASA Information Power Grid

- TeraGrid - Distributed Terascale Facility

- GriPhyN - Grid Physics Network

- iVDGL - International Virtual Data Grid Laboratory

- BIRN - Biomedical Informatics Research Network

- PPDG - Particle Physics Data Grid

- DATATAG - Trans-Atlantic Grid Testbed

- NEES - Network for Earthquake Engineering Simulation

- AstroGrid - UK Virtual Astronomy

- sciDAC - DOE Science Grid

- Nauka Grid - Russia Science Grid

- NorduGrid - Norway Science Grid

- APGrid - Asia Pacific Grid 
Grid Technology at a Glance 
Grid Technology at a Glance 


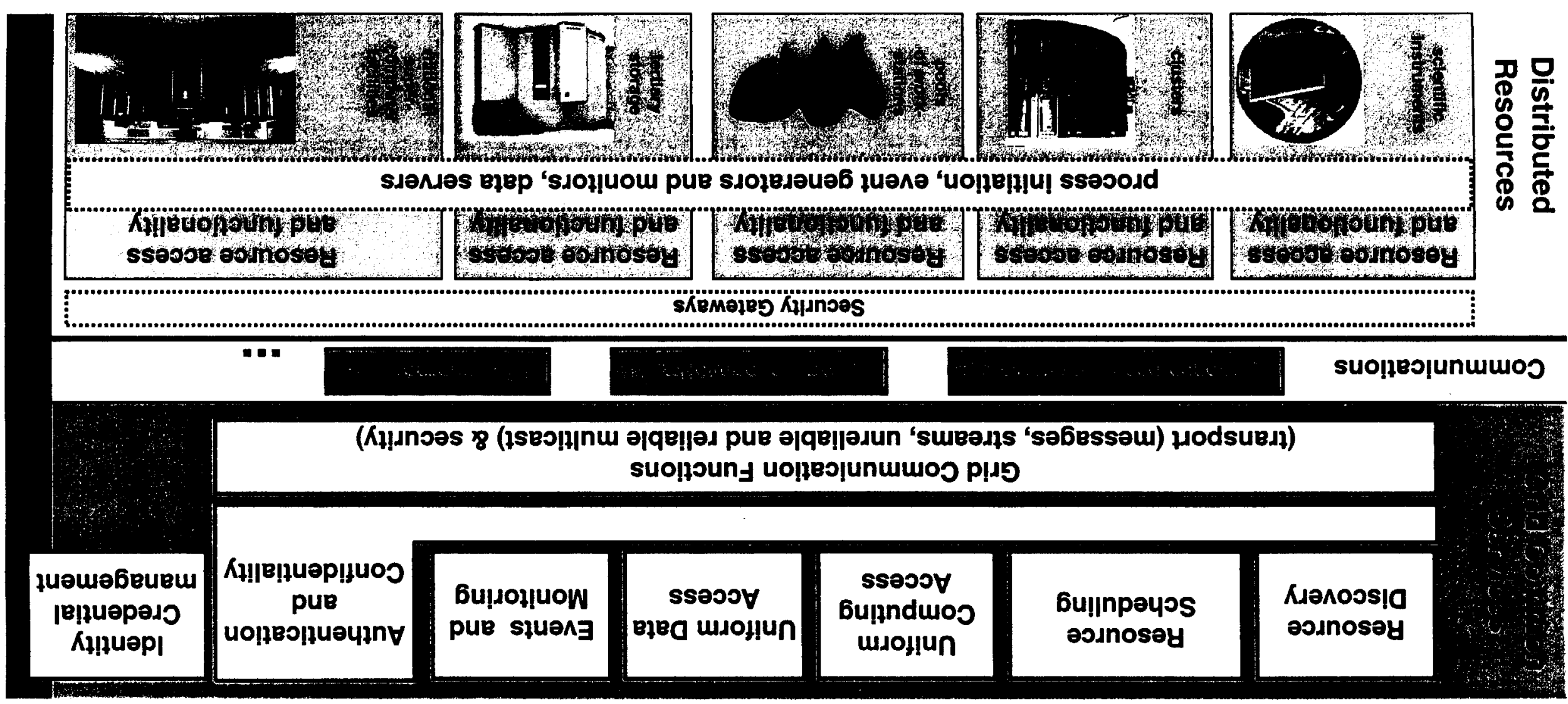

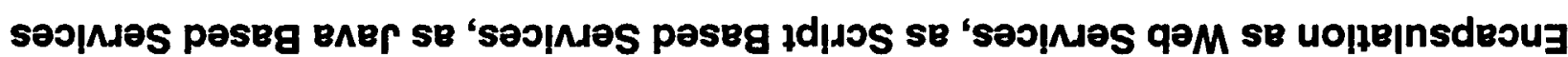

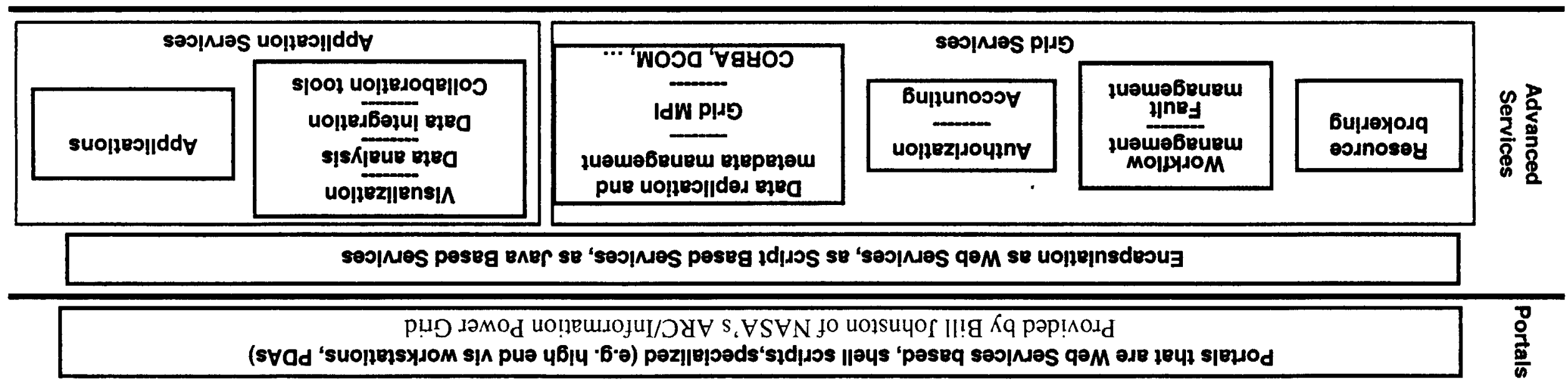



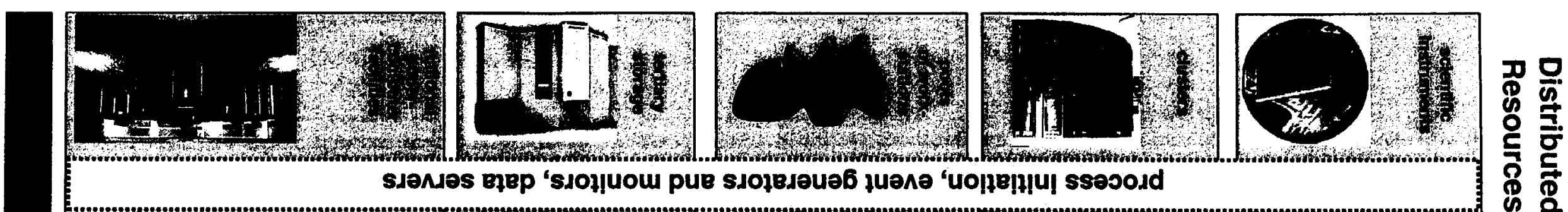

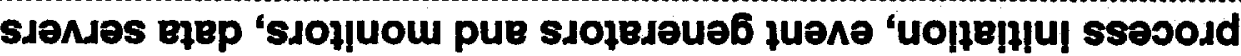
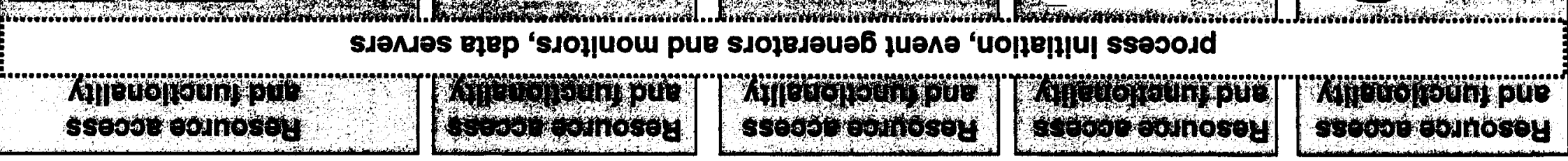

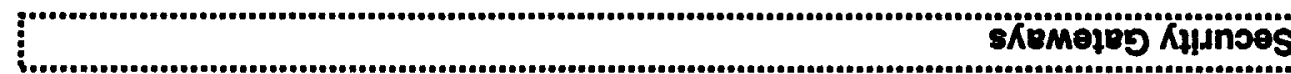

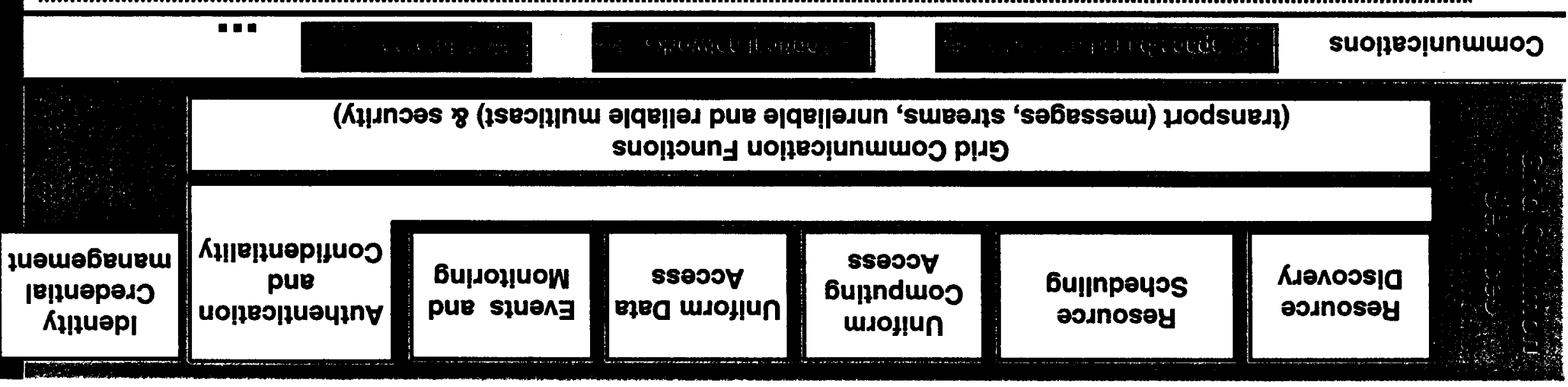

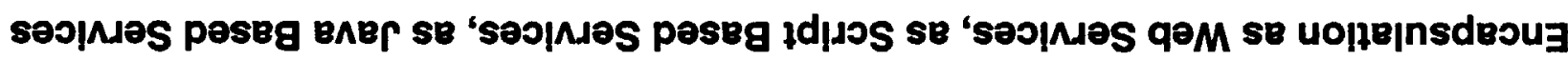

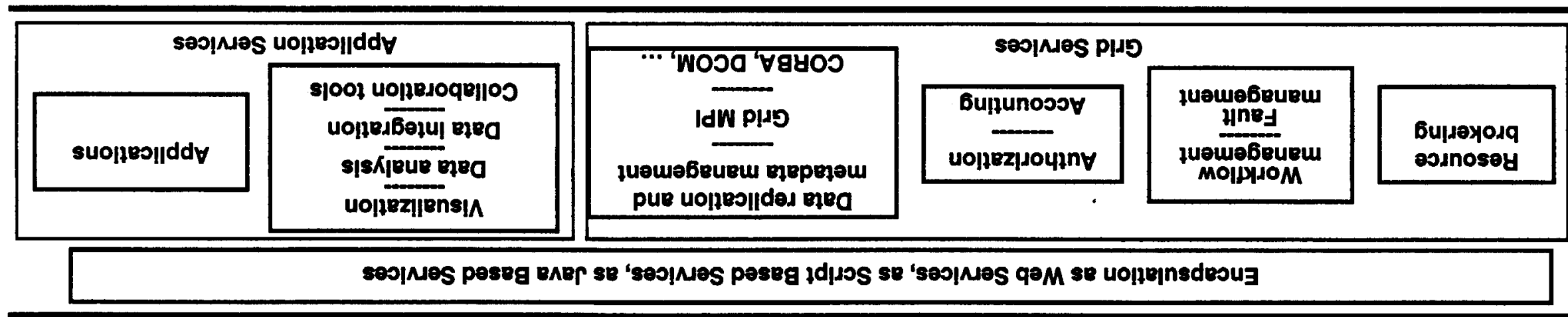

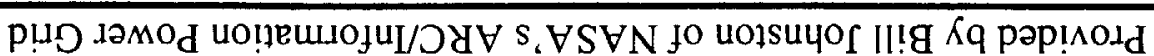

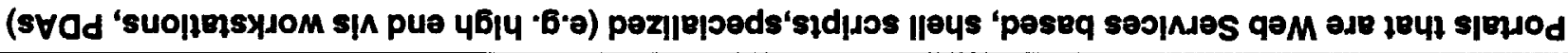


Space-based Science Portal Grid Concept 


\section{Space-based Science Portal Grid Concept}




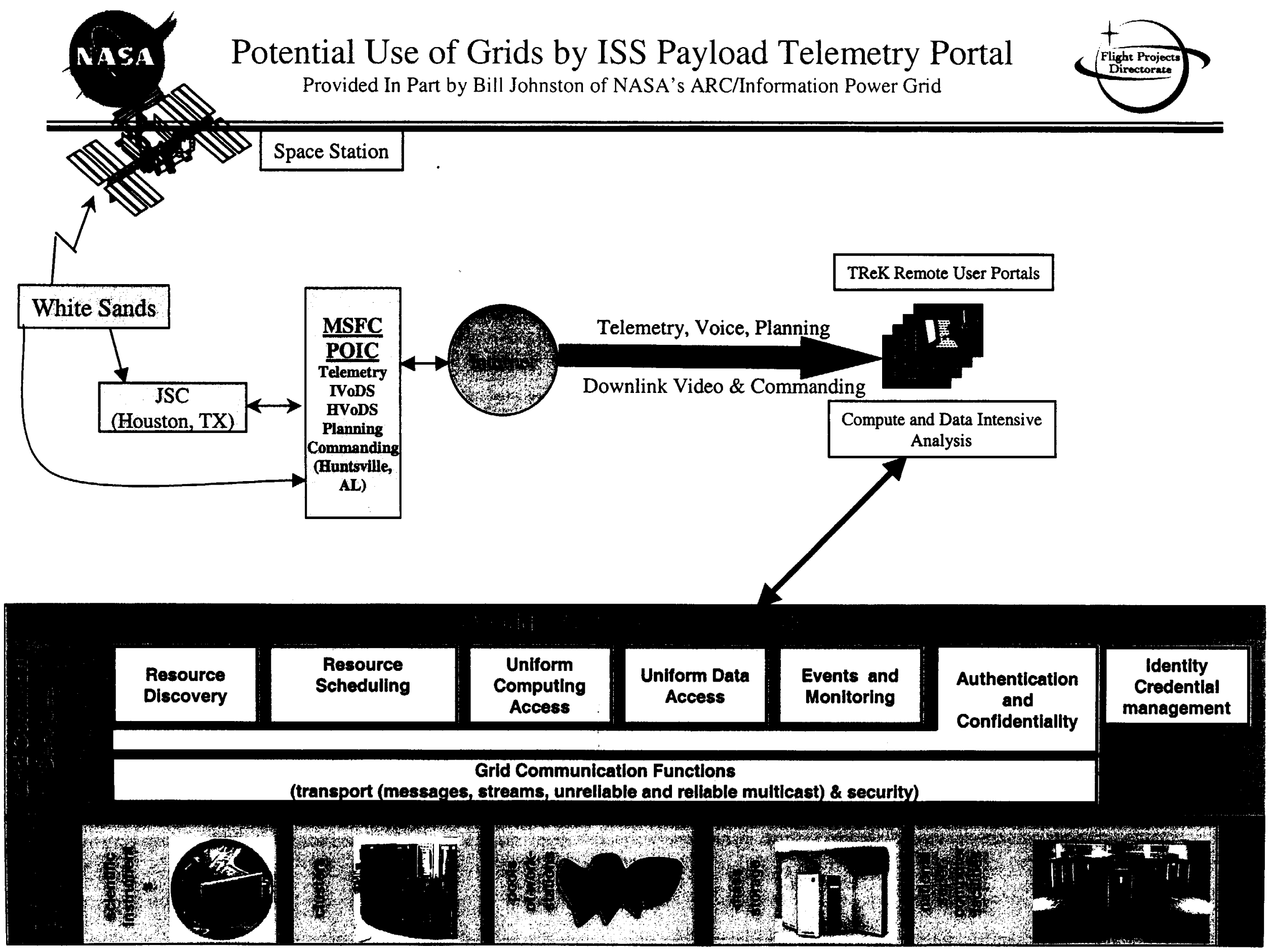



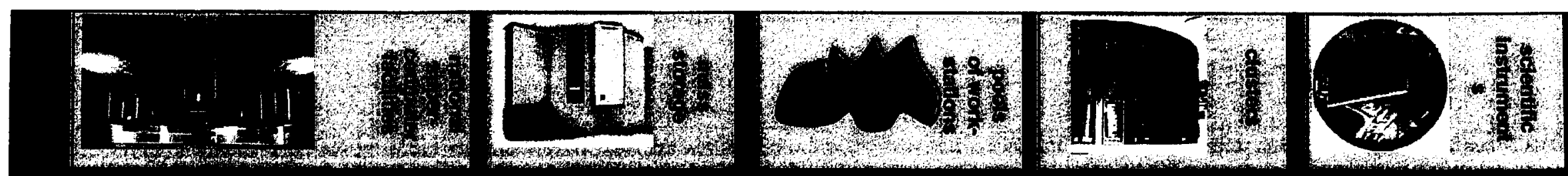

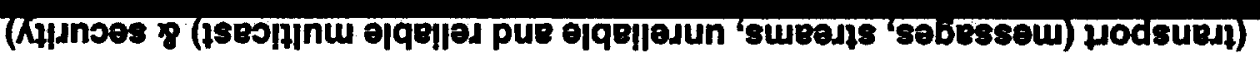

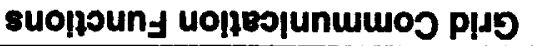

Iนจนจ6อนอน |еричеpedo Кұ\}uอp|

\section{(1)}

\begin{tabular}{|c|c|c|c|c|c|}
\hline 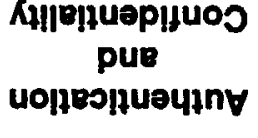 & $\begin{array}{l}\text { 6ujpofuow } \\
\text { pus squang }\end{array}$ & $\begin{array}{c}\text { sseosy } \\
\text { mpg usolun }\end{array}$ & $\begin{array}{l}\text { ssosoy } \\
\text { bujnduos } \\
\text { usolyun }\end{array}$ & $\begin{array}{c}\text { Bul|npeyos } \\
\text { osunosey }\end{array}$ & $\begin{array}{l}\text { Kienossid } \\
\text { eoinosey }\end{array}$ \\
\hline
\end{tabular}
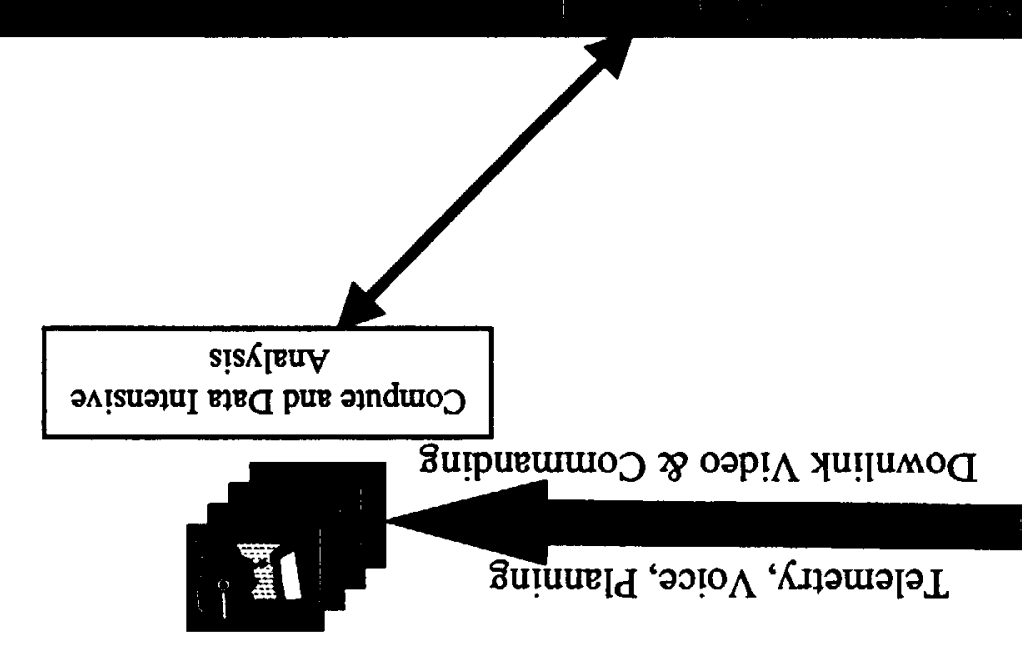

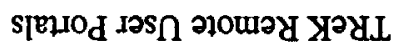

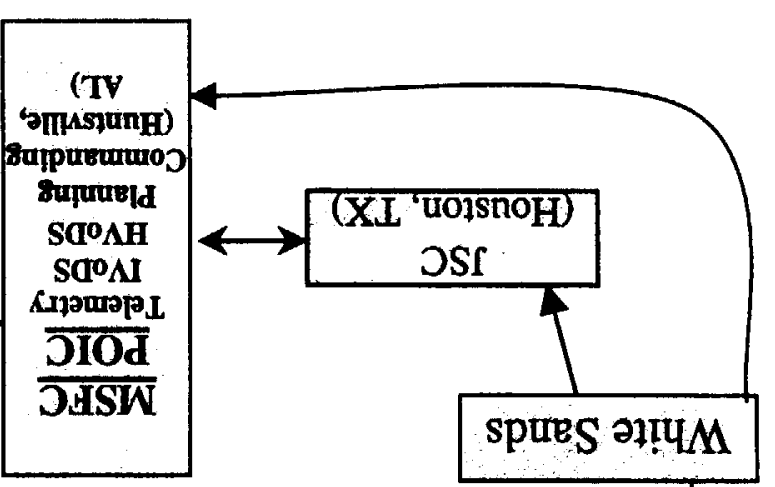

uome1S วoedS

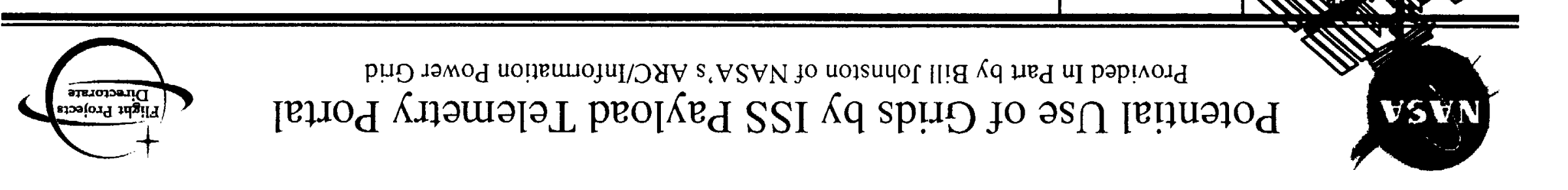




\section{Ops Services and the Necessary Technologies with Possible Existing Solutions by Component}

Services and (Potential Solutions) by Grid Portal Component:

\section{Component}

1. Security Component

- PKI
Potential Solution

(Imbedded at the system level)

2. Payload/Experiment Control

- Telemetry processing, distribution

EHS

- Command processing

EHS

- Database Services

EHS

- Display Services

EHS

- Telemetry acquisition and distribution

PDSS

- Payload planning

PPS

- Voice Distribution

3. Network Component

4. Remote User/Instrument Portal Component

- Mission voice including air to ground and space to ground links

- ISS Video

- Commanding and command data basing

- Telemetry processing and data display

- Planning and re-planning

5. Educational and Public Outreach Component

IVoDS

Abilene

IVoDS

IViDS requires development

TReK, Web

TReK, Web

Web, Rose \& other TBD systems

(EZStream®) 


\section{Ops Services and the Necessary Technologies with Possible Existing Solutions by Component}

Services and (Potential Solutions) by Grid Portal Component:

\section{Component}

1. Security Component

- PKI

2. Payload/Experiment Control

- Telemetry processing, distribution

- Command processing

- Database Services

- Display Services

- Telemetry acquisition and distribution

- Payload planning

- Voice Distribution

3. Network Component

4. Remote User/Instrument Portal Component

- Mission voice including air to ground and space to ground links

- ISS Video

- Commanding and command data basing

- Telemetry processing and data display

- Planning and re-planning

5. Educational and Public Outreach Component
Potential Solution

(Imbedded at the system level)

EHS

EHS

EHS

EHS

PDSS

PPS

IVoDS

Abilene

IVoDS

IViDS requires development

TReK, Web

TReK, Web

Web, Rose \& other TBD systems

(EZStream®) 


\section{Conclusions and Follow On}

- Conclusions:

- Grid technology development is well funded, backed by major computing and networking vendors and has been or currently is being implemented worldwide

- MSFC's ISS Payload Operations Integration Center is well positioned to participate in a prototype Space-based Science Operation Grid Portal by leveraging TReK, IVoDS and ERIS capabilities into the Portal services

- Network measurements is on-going as of July 2002

- Introducing real-time high end performance processing and collaboration tools into the Space-based science operation that may have significant application for future operations and science

- Follow On:

- Develop a prototype of a Space-based Science Operations Portal and Grid for ISS payloads collaborating with MSFC/POIC, UAH and ARC

- Determine the applicability by polling the science community of introducing a real-time high end processing capability

- Participate in the various Grid development initiatives 


\section{Conclusions and Follow On}

- Conclusions:

- Grid technology development is well funded, backed by major computing and networking vendors and has been or currently is being implemented worldwide

- MSFC's ISS Payload Operations Integration Center is well positioned to participate in a prototype Space-based Science Operation Grid Portal by leveraging TReK, IVoDS and ERIS capabilities into the Portal services

- Network measurements is on-going as of July 2002

- Introducing real-time high end performance processing and collaboration tools into the Space-based science operation that may have significant application for future operations and science

- Follow On:

- Develop a prototype of a Space-based Science Operations Portal and Grid for ISS payloads collaborating with MSFC/POIC, UAH and ARC

- Determine the applicability by polling the science community of introducing a real-time high end processing capability

- Participate in the various Grid development initiatives 
TOP ISSUES

07/24/02

\begin{tabular}{|c|c|c|c|}
\hline & ISSUES & ACTION WE'RE TAKING & WAYS DA01 COULD HELP* \\
\hline 1 & $\begin{array}{l}\text { Biological Research Project } \\
\text { Cost/Schedule Performance }\end{array}$ & $\begin{array}{l}\text { Monitoring Headcount } \\
\text { Weekly } \\
\text { Cost currently on plan. } \\
\text { Contractor reserves to } \\
\text { cover additional software } \\
\text { load/regression test. } \\
\text { - } \begin{array}{l}\text { Continued focus on results } \\
\text { of Internal Baseline } \\
\text { Review. }\end{array}\end{array}$ & \\
\hline 2 & $\begin{array}{l}\text { Contract consolidation } \\
\text { Projectization activities with } \\
\text { the ISS Program have created } \\
\text { an uncertain future work plan } \\
\text { for FD31, resulting in } \\
\text { increased employee work } \\
\text { dissatisfaction and stress }\end{array}$ & $\begin{array}{l}\text { Communicating openly } \\
\text { with employees, the status } \\
\text { of consolidation activities, } \\
\text { and negotiations on future } \\
\text { tasks } \\
\text { Attended meetings at JSC } \\
\text { to discuss plan for } \\
\text { consolidation with Boeing } \\
\text { \& ISS Payloads Office. } \\
\text { Provided fact-finding } \\
\text { questions on the ISS } \\
\text { Payloads Integration } \\
\text { Contract (IPIC) }\end{array}$ & $\begin{array}{l}\text { Continue to communicate Center } \\
\text { skill needs and future work plans }\end{array}$ \\
\hline 3 & $\begin{array}{l}\text { Potential design modification } \\
\text { to BRP to incorporate dual } \\
\text { seal quick disconnects }\end{array}$ & $\begin{array}{l}\text { Working with Payloads } \\
\text { Safety Panel to determine } \\
\text { whether a requirement } \\
\text { exists to have dual seal } \\
\text { quick disconnects (would } \\
\text { be considered a new } \\
\text { requirement to the } \\
\text { Projects) }\end{array}$ & \\
\hline 4 & $\begin{array}{l}\text { Pending Internal Thermal } \\
\text { Control System (ITCS) fluid } \\
\text { change-out }\end{array}$ & $\begin{array}{l}\text { Working with Boeing to } \\
\text { determine impacts to } \\
\text { hardware development } \\
\text { schedules in event of } \\
\text { PRCU implementation } \\
\text { of fluid change-out }\end{array}$ & \\
\hline
\end{tabular}

